CASE REPORT

\title{
An Unusual Masquerader Residing in the Lungs
}

\author{
Isabella Princess ${ }^{1}$, Anila Mathan ${ }^{2}$, Shanmugaraj $T^{3}$, Suresh Kumar ${ }^{4}$
}

\begin{abstract}
Parasitic infections of the lungs are almost always rare as the number of causative parasites is limited. There is an equal balance between protozoans and helminths causing lung infections. Most of the times, these infections are only seen in immunocompromised individuals. Among the helminths causing infection in this group of patients, Strongyloides stercoralis is associated with hyper infection syndrome. Most of these infections go unnoticed as they are not looked for carefully due to non-suspicion of the same. Development or exacerbation of symptoms is common in acute Strongyloidiasis. In such cases, the mortality rate is quite high. We present a case of pulmonary Strongyloidiasis who presented with acute exacerbation of COPD on corticosteroid therapy in whom the outcome was fatal. J Microbiol Infect Dis 2017; 7(1): 51-53
\end{abstract}

Keywords: Strongyloidiasis, Strongyloides stercoralis, bronchiectasis, COPD

\section{INTRODUCTION}

Very few parasites are associated with respiratory tract infections. Although most of these parasites produce cystic or space occupying lesions; few do not have a specific clinical presentation, thereby producing diffuse infiltrates in the lungs [1]. Among the parasites infecting the lungs, focal lesions are commonly seen in Hydatid disease and Dirofilariasis. Consolidation / pleural effusion are usually seen in Paragonimiasis, Amoebiasis. However, diffuse pulmonary infiltrates are seen in Ascariasis, Hook worm infection, Toxocariasis. Alveolar/ interstitial changes are seen in Strongyloidiasis [1]. Although rare in involving the lungs, Strongyloides infection of the lungs have been reported from countries like India, Turkey etc. [2,3]. Though it mainly occurs in immunocompromised individuals, it has been reported from immune competent individuals as well [3].

We present a case of Strongyloides infection of the lungs in an immunocompromised host on long term corticosteroid therapy. The clinical findings were unusual and mimicked other infectious diseases of the lungs which made the diagnosis difficult initially.

\section{CASE}

A 56 year-old male patient who hails from a town near Chennai, India presented with complaints of breathlessness and cough with expectoration for three days. Sputum was purulent, greyish brown in color in copious amount. The patient was on regular medication for bronchiectasis, COPD, type 2 diabetes mellitus and systemic hypertension. Few months ago he was admitted for acute exacerbation of COPD and community acquired pneumonia and was treated with appropriate antibiotics and respiratory support. $\mathrm{He}$ was on long term corticosteroid therapy for bronchial asthma, COPD and bronchiectasis. During the present admission, due to worsening dyspnea, he was put on non-invasive ventilation. Chest X-Ray showed increasing diffuse infiltrates. CT Chest showed multiple focal areas of consolidation, cavities, and ground glass opacities in both lungs involving all the lobes with diffuse areas of bronchiectasis.

Laboratory investigations revealed abnormal absolute eosinophil count of 85 cells/cu.mm. However the WBC count was elevated $(21.88 \mathrm{x}$ $10^{3} / \mathrm{mm}^{3}$ ). No anemia was noted (Hemoglobin= $13.0 \mathrm{gm} \%)$.Routine stool examination was

\footnotetext{
${ }^{1}$ Department of Microbiology, ${ }^{2}$ Department of Hematology and Transfusion Medicine, ${ }^{3}$ Department of Internal Medicine,

${ }^{4}$ Department of Infectious Diseases; Apollo Speciality Hospitals, Vanagaram, Chennai, India

Correspondence: Dr. Isabella Princess, Department of Microbiology, Apollo Specialty Hospitals, Vanagaram Branch, Ayanambakkam, Chennai, India, E-mail: isadear@gmail.com Received: 10 November 2016, Accepted: 31 December 2016

Copyright (C JMID / Journal of Microbiology and Infectious Diseases 2017, All rights reserved
} 
negative for helminthic ova and protozoan cysts. HIV antibodies were negative.

Upon suspicion of pulmonary tuberculosis due to major involvement of the upper lobes of the lungs, sputum samples were sent for acid fast stain and culture. No acid fast bacilli were found. After two days, a repeat sputum sample was sent for fungal culture and $\mathrm{KOH}$ mount. On $\mathrm{KOH}$ mount, filariform larvae measuring approximately $610-620 \mu \mathrm{m} \times 15-16 \mu \mathrm{m}$ in size resembling that of Strongyloides stercoralis were noticed. To confirm the same, Lugol's iodine wet mount was prepared. This helped in delineating the internal structures of the larvae clearly. A prominent double bulb esophagus was seen, tail tip was blunt, which was strongly suggestive of Strongyloidiasis. Gram stain and acid fast stain were prepared from the same sample. Larvae were also observed in both these stained smears (Figure 1 and 2).

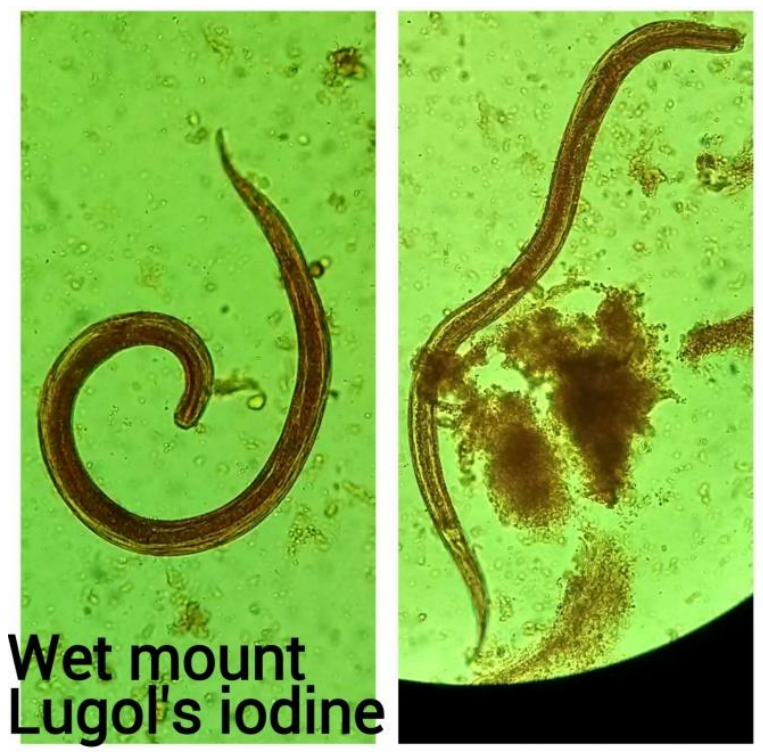

Figure 1. Wet mount Lugol's iodine.

Patient was immediately started on Tab. Ivermectin $12 \mathrm{mg}$. Two days later, due to severe tachypnea and restlessness, he was intubated. An endotracheal aspirate was sent which also showed motile larvae of Strongyloides stercoralis. Aerobic culture of sputum and endotracheal aspirate grew normal flora of the upper respiratory tract. $\mathrm{He}$ had severe bronchospasm which was treated with nebulized bronchodilators. Patient became hemodynamically unstable and had hypoxic cardiac arrest for which inotropes were started. There was persistent bronchospasm. Patient went into cardiac arrest and despite all resuscitative efforts; patient could not be revived and was declared dead.
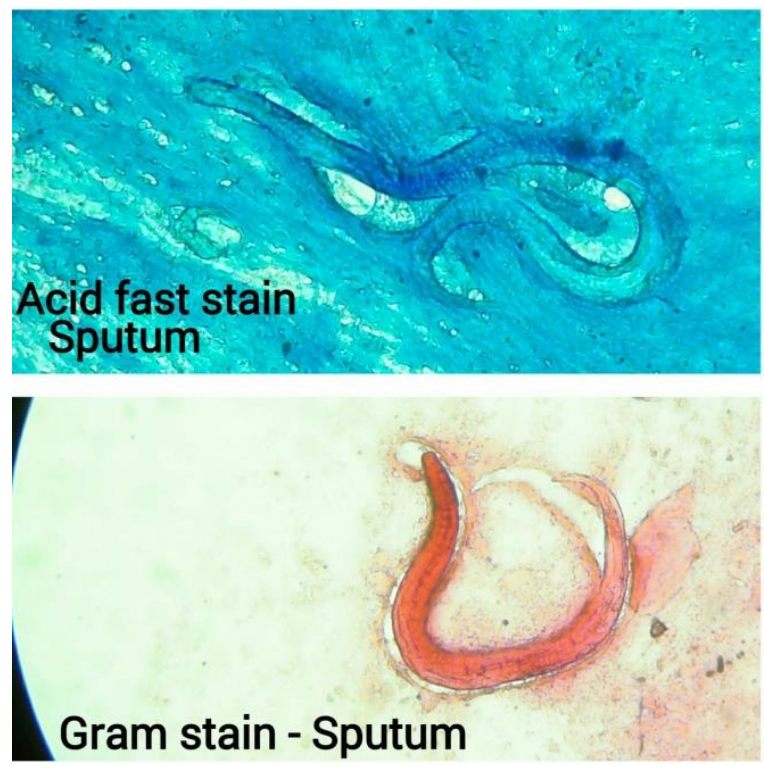

Figure 2. The parasite from sputum.

\section{DISCUSSION}

In the recent past, there is a surge in the number of parasitic infections involving immunocompromised individuals. Most of these reports including ours arise from tropical and sub-tropical countries around the globe [2-4]. Most of these patients are immunocompromised and majority are on corticosteroid therapy [5]. Our patient was also on long term corticosteroids for bronchial asthma and COPD. The reasons for corticosteroid therapy among patients with Strongyloides infection as observed in other studies were similar to our patient as for COPD, asthma, lung fibrosis [6]. Patients with pulmonary involvement of Strongyloides infection have symptoms such as cough, dyspnea, wheezing and hemoptysis. This is due to the larvae reaching the lungs [7]. Our patient had similar symptoms for a long duration of time. Although hyperinfection with Strongyloides stercoralis is common among patients with HIV infection, our patient tested negative for HIV antibodies.

It is not uncommon to isolate bacterial enteric flora from sputum of patients with Strongyloides 
lung infections suggestive of migratory stage of the larvae from the gut into the pulmonary capillaries [8]. However, that was not the case in our patient as we did not isolate any bacteria from his sputum nor endotracheal aspirate. This explains the reason why the larvae were not detected in stool as the load could be low or that the larvae were in the migratory stage rather than in the stage of dissemination [1]. As a supportive evidence to larvae being absent in stool, the patient did not give a history of diarrhea.

Absolute eosinophil count elevation is usually suggestive of chronic parasitic infection [1]. In our patient, eosinophilia was not observed, which suggests a possibility that he had acute Strongyloidiasis. Pulmonary involvement could be fatal even after treatment with Ivermectin [2]. This was the similar situation in our case as he succumbed to the illness although he was on Ivermectin. Various authors around the world have suggested a significant association between coexisting lung disease and strongyloidiasis [9]. Our patient's history was also suggestive of an association of strongyloidiasis with coexisting lung disease. As his chest $X$ ray suggested diffuse infiltrates, it is strongly suggestive of Strongyloidiasis as this is one among the common chest $\mathrm{X}$ ray findings among $17 \%$ and $45 \%$ of these patients [10]. CT findings in our patient showed ground glass opacities, consolidation and cavitation. These could possibly be due to chronic bronchiectasis. However, ground glass opacities on CT chest were observed in a case of pulmonary Strongyloidiasis reported by authors from Turkey [3].

In conclusion, this particular case is educative in many ways as the clinical features observed were quite diverse and masquerading many other conditions such as fungal and mycobacterial infections of the lungs. This could be a learning point to look for parasites in respiratory samples as the number of immunocompromised individuals is increasing in the recent past due to corticosteroid therapy. Parasitic lung infections may not always have a typical presentation on imaging studies. This makes the definitive diagnosis difficult and cumbersome. Hence, visualization of these parasites in clinical specimens still remains the gold standard and confirmatory of the same.
Awareness on wearing protective footwear should be emphasized as these geohelminthic larvae are found in soil and gain access by penetrating intact skin of humans. Deworming should be considered on a regular basis not only for children, but also for adults.

Declaration of conflicting interests: The authors declared no conflicts of interest with respect to the authorship and/or authorship of this article.

Funding: The authors received no financial support for the research and/or authorship of this article.

\section{REFERENCES}

1. Kunst H, Mack D, Kon OM, Banerjee AK, Chiodini P, Grant A. Parasitic infections of the lung: a guide for the respiratory physician. Thorax 2010; 66(6):528-536.

2. Tadepalli K, Sagar K. A case of masquerading bronchopneumonia. Lung India 2014; 31(2):16163.

3. Canan D, Mine G, Ayse O, et al. Pulmonary Strongyloides stercoralis infection. Respir Med Case Rep 2014; 11:12-15.

4. Sowmya N, Jeevan M, Swathi G, Mohanakrishnan K. A Case Report of Strongyloidiasis associated with Giardiasis in a patient with renal calculi from a Tertiary Care Center in South India. J Glob Infect Dis $2014 ; 6(3): 137$

5. Dora B, Ana R, Andrea A, et al. Severe strongyloidiasis: a systematic review of case reports. BMC Infect Dis 2013; 13:78.

6. Kim J, Joo HS, Ko HM, Na MS, Hwang SH, Im JC: A case of fatal hyperinfective strongyloidiasis with discovery of autoinfective filariform larvae in sputum. Korean J Parasitol 2005;43(2):51-55.

7. Vijayan VK. How to diagnose and manage common parasitic pneumonias. Curr Opin Pulm Med 2007; 13:218-24.

8. Rathor N, Khillan V, Sarin SK. Strongyloides stercoralis hyperinfection in patient with autoimmune hepatitis and purpura fulminans. Indian J Crit Care Med 2016; 20:52-4.

9. Davidson RA. Infection due to Strongyloides stercoralis in patients with pulmonary disease. South Med J 1992; 85:28-31.

10. Mokhlesi B, Shulzhenko O, Garimella PS, Kuma L, Monti C. Pulmonary strongyloidiasis: the varied clinical presentations. Clin Pulm Med 2004; 11: 6. 